\title{
Die proximale öffnende Tibiaosteotomie zur Behandlung der Varusgonarthrose
}

\author{
Mellany Galla, Philipp Lobenhoffer
}

\section{Zusammenfassung}

Die valgisierende Tibiakopfosteotomie in öffnender Technik („open wedge“) ist ein geeignetes Verfahren zur Behandlung der medialen Gonarthrose bei vorliegender Varusdeformität. Das Ziel des Verfahrens ist die Entlastung des medialen Kniegelenkkompartiments durch Verschiebung der mechanischen Tragachse nach lateral. Die in diesem Beitrag vorgestellte medial öffnende OP-Methode bietet im Gegensatz zur lateral schließenden Technik den Vorteil, dass eine Muskelablösung und die Schädigung des N. peronaeus vermieden werden kann. Es wird eine biplanare Osteotomie im $100^{\circ}$-Winkel durchgeführt. Die Osteotomie wird unter Erhalt einer lateralen Knochenbrücke langsam aufgespreizt und das Korrekturergebnis wird mit einem winkelstabilen internen Plattenfixateur (TomoFix ${ }^{\mathrm{TM}}$ ) fixiert. Die beschriebene Technik erlaubt in den meisten Fällen die Durchführung der öffnenden Tibiaosteotomie, ohne dass der Osteotomiespalt mit Spongiosa oder Knochensubstitut aufgefüllt werden muss.

\section{The High Tibial Open Wedge Osteotomy}

The open-wedge high tibial osteotomy is an effective treatment modality for medial gonarthrosis by transferring the mechanical leg axis from the medial to the lateral compartment. In contrast to the lateral closed-wedge technique, the open-wedge procedure avoids detachment of extensor muscles and lesions of the superficial peroneal nerve. A biplanar $100^{\circ}$ angulated osteotomy is performed and the osteotomy ends $5-10 \mathrm{~mm}$ medial of the lateral cortex. The osteotomy is opened gradually with a spreader and fixed with a TomoFix ${ }^{\mathrm{TM}}$ plate fixator. In most cases neither autogenous cancellous bone nor bone substitution are needed for filling the osteotomy gap.

\section{Einleitung}

Die Abweichung der mechanischen Beinachse von der physiologischen Norm gilt als präarthrotische Veränderung des Kniegelenks mit belastungsabhängigen Schmerzen im überlasteten Gelenkkompartiment. Die physiologischen Achsenverhältnisse können mit kniegelenknahen Umstellungsosteotomien wiederhergestellt werden. Die häufigste Fehlstellung des Beines in der Frontalebene ist die Varusdeformität,

OP-JOURNAL 2011; 27: 254-259

(C) Georg Thieme Verlag KG Stuttgart · New York DOI http://dx.doi.org/10.1055/s-0031-1280445 bei der die Belastungstragachse nicht durch das Kniegelenkzentrum verläuft, sondern durch das mediale Kompartiment (Abb.1). Dies resultiert in einer medialen Gonarthrose.

Die proximale valgisierende Tibiakopfosteotomie ist ein bewährtes Behandlungsverfahren der medialen Gonarthrose [2]. Die mechanische Beinachse (sog. Mikulicz-Linie) wird nach lateral korrigiert verlagert und der mediale Gelenkabschnitt entlastet [3].

Die Wiederherstellung der normalen Achsenverhältnisse kann die endoprothetische Versorgung bei jungen und körperlich aktiven Patienten verhindern oder hinauszögern und führt zu guten Ergebnissen [2]. Hierzu sind prinzipiell verschiedene OP-Techniken an der proximalen Tibia möglich:

- medial öffnende Osteotomien („open wedge“),

- lateral schließende Osteotomien („closed wedge“),

- domförmige oder giebelförmige Osteotomien (Pendelosteotomien).

Pendelosteotomien sind technisch schwierige und anspruchsvolle Eingriffe.

Bei der lateral schließenden OP-Methode wird ein Knochenkeil mit einer lateralen Basis entnommen. Durch das Schließen des dadurch entstandenen Spaltes wird die Beinachse nach lateral korrigiert. Bei diesem Vorgehen müssen jedoch das proximale Tibiofibulargelenk gelöst und die Extensorenmuskulatur abgelöst werden. Ferner besteht ein hohes Risiko einer Schädigung des N. peronaeus [1].

Bei der medial öffnenden Technik („open wedge“) werden diese Risiken umgangen. Die medialseitige Osteotomie wird langsam geöffnet, bis die Achsenkorrektur erreicht ist. Zur Fixation des Ergebnisses empfiehlt sich ein spezieller winkelstabiler Plattenfixateur, der sich durch eine hohe biomechanische Stabilität auszeichnet. Ein Vorteil der öffnenden gegenüber der schließenden Methode ist die Möglichkeit des intraoperativen „fine-tunings“.

Die Achse kann intraoperativ kontrolliert und durch weiteres Öffnen oder Schließen des Osteotomiespalts ggf. justiert werden.

Gleichzeitig ist eine Behandlungsmöglichkeit von Bandinstabilitäten durch Veränderung der dorsalen Neigung des tibialen Plateaus („tibial slope“) gegeben. 


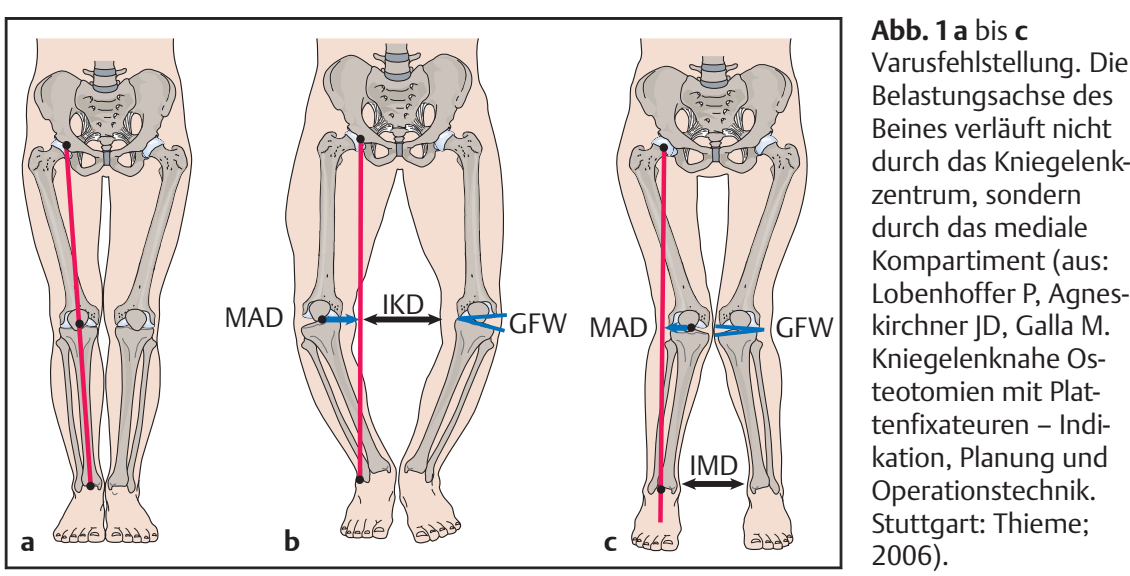

Tab. 1 Indikationen der valgisierenden Umstellungsosteotomie.

- unikompartimentelle mediale Gonarthrose

\begin{tabular}{l}
\hline - Varusdeformität der unteren Extremität \\
\hline - Patientenalter unter 65-70 Jahre \\
\hline - Patienten mit körperlichem Aktivitäts- \\
anspruch \\
\hline - präoperativer Bewegungsumfang des \\
Kniegelenks von mindestens E/F 0/10/ \\
$120^{\circ}$ \\
\hline - als Begleiteingriff zu Knorpel- und \\
Meniskuseingriffen (s. Text) \\
\hline - als valgisierend-flektierende oder -ex- \\
tendierende Tibiakopfosteotomie bei \\
zusätzlichen Bandinstabilitäten (s. Text)
\end{tabular}

\section{OP-Planung}

Klinische Untersuchung und Röntgendiagnostik

Neben der Anamnese muss eine sorgfältige präoperative klinische Untersuchung erfolgen. Diese umfasst die Überprüfung des Bewegungsumfangs und des Bandapparats des Kniegelenks. Die Haut- und Weichteilverhältnisse müssen inspiziert werden und reizlos sein, vorbestehende Operationsnarben müssen ggf. beim $\mathrm{Zu}-$ gangsweg berücksichtigt werden.

Die präoperative radiologische Diagnostik umfasst Röntgenaufnahmen des Kniegelenks in 3 Ebenen sowie eine Ganzbeinaufnahme im Stehen.

Eine Belastungsaufnahme des Kniegelenks in $45^{\circ}$ Flexion (sog. Rosenberg-Aufnahme) und eine MRT-Untersuchung sind nicht obligat, können jedoch Aufschluss über das Ausmaß der Knorpel-
Tab. 2 Kontraindikationen der valgisierenden Umstellungsosteotomie

- Arthrose bzw. dritt- bis viertgradige Knorpelschäden im lateralen Kompartiment

\begin{tabular}{l} 
- Außenmeniskusverlust \\
\hline - starkes Übergewicht
\end{tabular}

- unzureichende Weichteilverhältnisse an der proximalen medialen Tibia

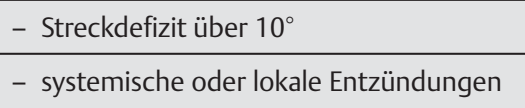

schädigung im medialen Gelenkkompartiment geben.

Anhand der Ganzbeinaufnahme wird präoperativ das erforderliche Korrekturausmaß bestimmt und die Höhe der Osteotomieöffnung ermittelt.

\section{Indikationen und Kontraindikationen}

Die Indikation zur valgisierenden öffnenden Tibiakopfumstellungsosteotomie ist die unikompartimentelle mediale Gonarthrose und eine Varusdeformität bei Patienten mit körperlichem Aktivitätsanspruch (Tab. 1). Das Patientenalter sollte nicht über 65-70 Jahre liegen. Das OP-Verfahren kann begleitend zu einer Mikrofrakturierung im betroffenen Kniegelenkabschnitt durchgeführt werden. Bei einer Ersatzplastik des vorderen Kreuzbands bei vorliegender Varusfehlstellung kann eine valgisierend-extendierende Umstellungsosteotomie der proximalen Tibia zu einer Achskorrektur und Verminderung der vorderen Schublade führen. Eine valgisierend-flektierende proximale Tibiaosteotomie führt zu einer Stabilisierung bei posterolateraler Kniegelenkinstabilität bei Varusmor-

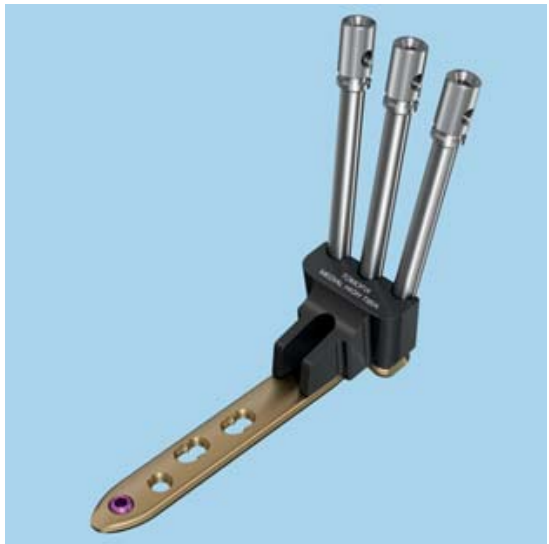

Abb. 2 TomoFixNGTM-Plattenfixateur mit vormontierten Bohrbüchsen und Distanzstopfen.

photyp. Der Bewegungsumfang des Kniegelenks sollte präoperativ mindestens $0-10-120^{\circ}$ für Streckung/Beugung betragen. Durch die o.g. gleichzeitige flektierende oder extendierende Komponente der Osteotomie können Streck- und Beugedefizite adressiert werden.

Als Kontraindikationen gelten starkes Übergewicht, dritt- bis viertgradige Knorpelschäden nach Outerbridge [5] bzw. eine manifeste Arthrose des lateralen Gelenkanteils, Außenmeniskusverlust und eine eingeschränkte Kniegelenkbeweglichkeit, insbesondere Streckdefizite über $10^{\circ}$ (Tab. 2). Der Eingriff sollte auf keinen Fall bei schlechten Weichteilverhältnissen im Operationsgebiet sowie bei akuten oder chronischen Entzündungszuständen vorgenommen werden.

\section{Instrumentarium}

Für den Eingriff in der von uns beschriebenen Technik wird folgendes Instrumentarium benötigt:

- 1 TomoFix ${ }^{\mathrm{TM}}$-Plattenfixateur sowie dazugehörige bi- und monokortikale Kopfverriegelungsschrauben,

- oszillierende Säge mit einem breiten Sägeblatt (ca. $90 \mathrm{~mm}$ Länge) und einem schmalen Sägeblatt (ca. $50 \mathrm{~mm}$ Länge),

- 2 Führungsdrähte,

- mehrere Flachmeißel(15-20 mm Breite),

- optional 1 Spreizmeißel,

- 1 Messlehre oder 1 steriles Lineal,

- 1 Arthrodesen- bzw. Osteotomiespreizer,

- Messstange zur Beurteilung der Beinachse. 


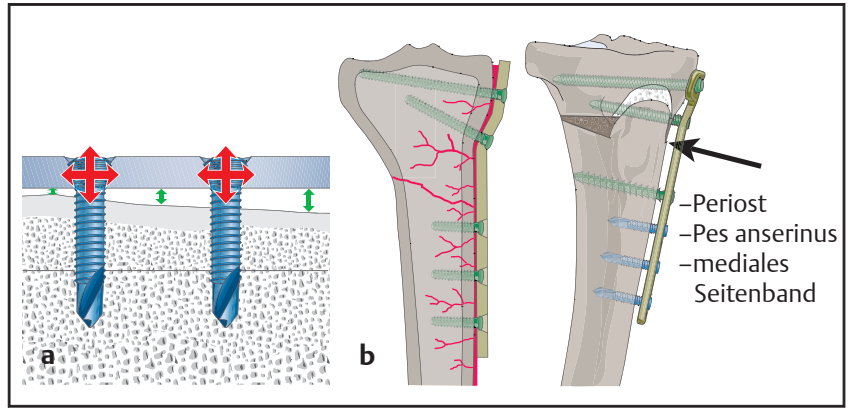

Abb. 3 a und b Durch die Distanzstopfen werden die periostale Durchblutung nicht beeinträchtigt und die Weichteile (Pes anserinus, mediales Seitenband) geschont (aus: Lobenhoffer P, Agneskirchner JD, Galla M. Kniegelenknahe Osteotomien mit Plattenfixateuren - Indikation, Planung und Operationstechnik. Stuttgart: Thieme; 2006).

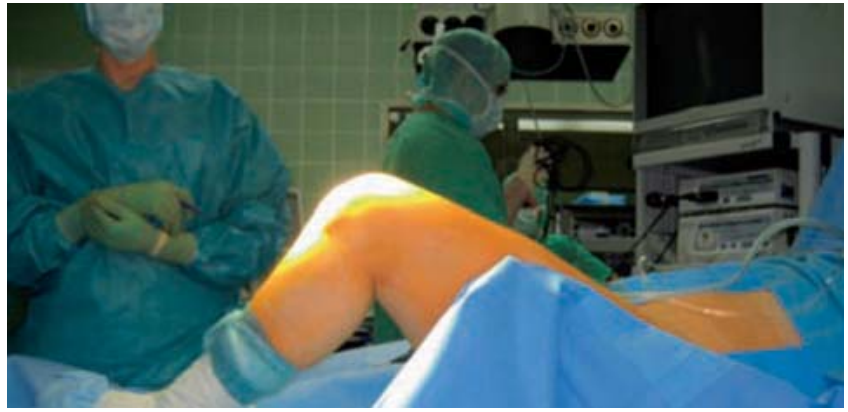

Abb. 4 Lagerung des Patienten.
Die Anwendung des TomoFix ${ }^{\mathrm{TM}}$-Plattenfixateurs erfordert genaue Kenntnisse des Implantats (Abb. 2) und der spezifischen winkelstabilen Verriegelungstechnik.

Die 5-mm-Kopfverriegelungsschrauben sind selbstschneidend (bikortikal, grün) und selbstschneidend/selbstbohrend (monokortikal, blau) und fungieren als Gewindebolzen. Von besonderer Wichtigkeit ist das exakte Eindrehen der Gewindebohrhülsen in das Schraubenloch vor dem Bohren, um ein Verkanten des Kopfgewindes beim Eindrehen der Schraube zu vermeiden. Die Schraubenköpfe werden mit einem Drehmomentschraubenzieher winkelstabil im Gewindeloch verriegelt. Im Längsschenkel des Implantats befinden sich LCP-Kombilöcher. Die vormontierten Distanzstopfen von $3 \mathrm{~mm}$ Länge dienen als Abstandshalter und limitieren den Druck der Plattenunterfläche auf das Periost. Drucknekrosen und Kompression der darunter liegenden Weichteile können somit vermieden werden und die periostale Blutversorgung wird auf diese Weise geschont (Abb. 3).

\section{Anästhesie und Lagerung}

Der Eingriff wird in Rückenlage entweder in Intubationsnarkose oder in rückenmarksnaher Anästhesie durchgeführt. Präoperativ wird eine systemische Antibiotikaprophylaxe als Single-Shot-Gabe appliziert.

An den OP-Tisch sollten eine Seitstütze und eine Fußrolle angebracht werden, damit das Bein problemlos sowohl in $90^{\circ}$-Flexion als auch in voller Streckung gelagert werden kann (Abb.4).

Die Abdeckung schließt den Beckenkamm mit ein, um eine intraoperative
Beurteilung der Beinachse und ggf. eine Spongiosaentnahme zu ermöglichen. Die Verwendung einer Oberschenkelblutsperre ist in der Regel nicht erforderlich, es kann aber eine sterile Blutsperre verwendet werden. Zur intraoperativen Durchleuchtungskontrolle wird der Bildverstärker auf der Gegenseite positioniert.

\section{OP-Technik}

Vor dem geplanten Eingriff wird in gleicher Narkose eine diagnostische Kniegelenkarthroskopie zur Inspektion der Knorpelverhältnisse durchgeführt.

Das laterale Kompartiment muss noch intakt, der Außenmeniskus sollte erhalten sein. Die Knorpelschäden im medialen Kompartiment werden exakt dokumentiert, instabile Knorpellappen werden entfernt und bedarfsweise kann eine Mikrofrakturierung erfolgen. Der Innenmeniskus wird nur bei mechanisch relevanter Rissbildung teilreseziert.

Der Eingriff beginnt bei aufgestelltem Bein in $90^{\circ}$-Beugung des Kniegelenks. Die anatomischen Landmarken (medialer Gelenkspalt, Oberrand des Pes anserinus, Verlauf des oberflächlichen Innenbands und Tuberositas tibiae) werden auf der Haut eingezeichnet. Der Hautschnitt verläuft vom Ansatz des Pes anserinus $6-8 \mathrm{~cm}$ nach dorsokranial ansteigend zur posteromedialen Ecke des Tibiakopfs (Abb.5). Die Subkutis wird durchtrennt und Sehnen des Pes anserinus werden dargestellt und mit einem Venenhaken oder Langenbeck-Haken nach distal gehalten. Nach dem Ablösen des distalen Anteils der oberflächlichen Innenbandfasern wird der Hinterrand der Tibia dargestellt. Ventral wird die mediale Begrenzung des Lig. patellae

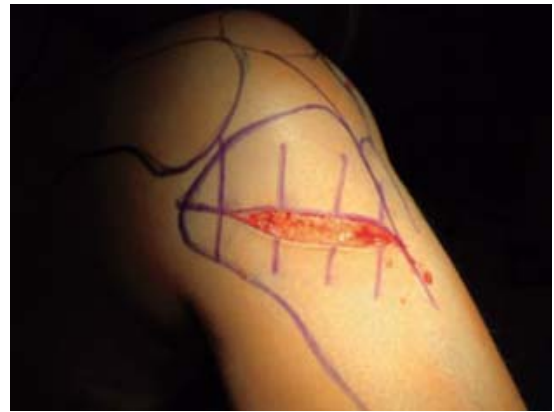

Abb. 5 Hautschnitt am medialen Tibiakopf.

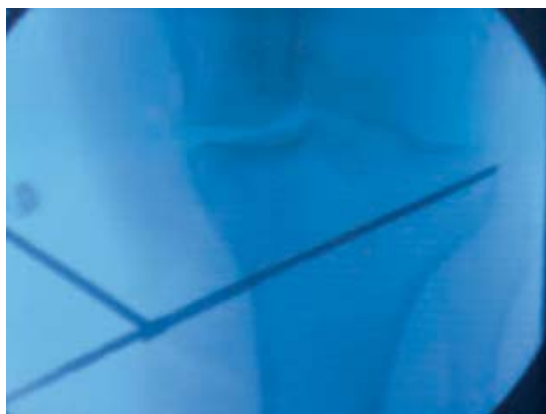

Abb. 6 Einbringen der 2 2,3-mm-Drähte zur Markierung des Osteotomieverlaufs mit Zielrichtung auf das obere Drittel des proximalen Tibiofibulargelenks. Die Drähte enden genau an der lateralen Tibiakortikalis.

identifiziert. Der Verlauf der Osteotomiehöhe und die Osteotomierichtung werden unter Durchleuchtungskontrolle durch Einbringen von 2 2,3-mm-Drähten markiert, wobei die Drähte auf das obere Drittel des proximalen Tibiofibulargelenks zielen und genau an der lateralen Tibiakortikalis enden (Abb.6). Zuerst wird der posteriore Draht platziert, der 2. Draht wird ca. $2 \mathrm{~cm}$ weiter ventral parallel zum 1. Draht eingebracht. Die Osteotomietiefe wird gemessen, wobei eine laterale Knochenbrücke von $10 \mathrm{~mm}$ Breite belassen werden soll. Unter Berücksichtigung dieser Knochenbrücke wird die Tiefe des erforderlichen Sägeschnitts auf dem Sägeblatt markiert. 


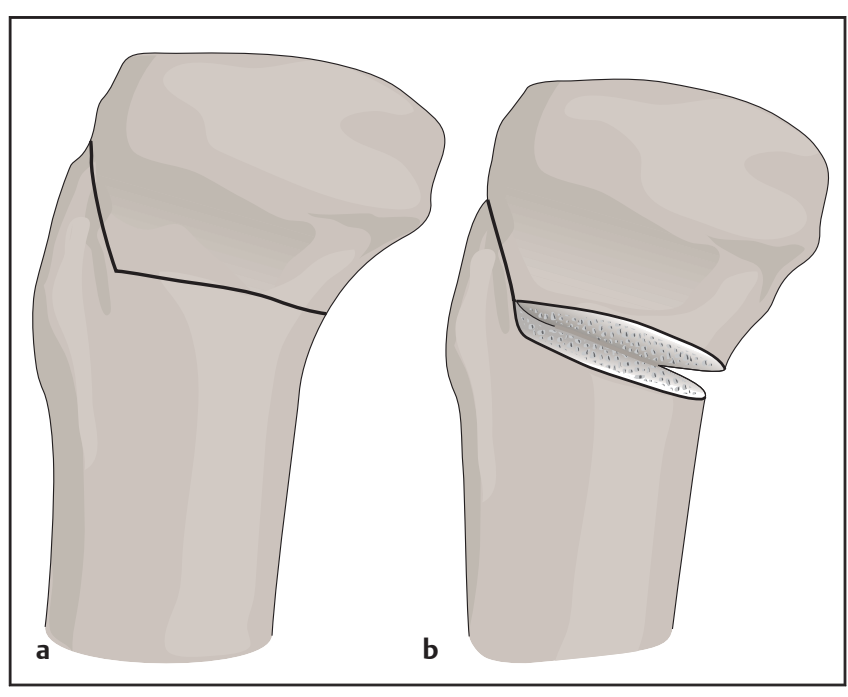

Abb. 7a und $\mathbf{b}$

Prinzip der biplanaren winkelförmigen Osteotomie (aus: Lobenhoffer $\mathrm{P}$, Agneskirchner JD, Galla M. Kniegelenknahe Osteotomien mit Plattenfixateuren - Indikation, Planung und Operationstechnik. Stuttgart: Thieme; 2006).

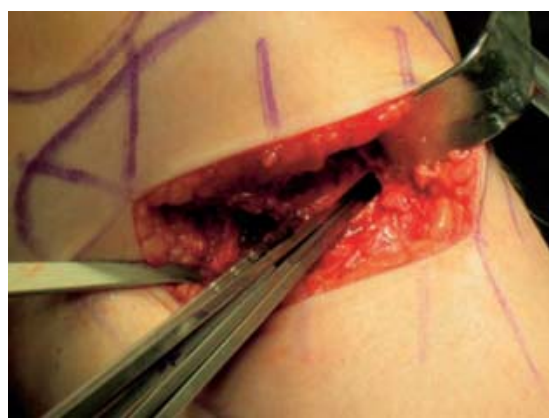

Abb. 8 Öffnen des Osteotomiespalts durch Einbringen mehrerer Flachmeißel.

Der vertikale Sägeschnitt verläuft im posterioren Zweidrittel der Tibia unterhalb der Führungsdrähte. Die ventrale Osteotomie verläuft hinter der Tuberositas tibiae nach kranial ansteigend und bildet zum vertikalen Schnitt einen Winkel von $100^{\circ}$ (Abb. 7). Die Breite dieses Tuberositassegments sollte mindestens $15-20 \mathrm{~mm}$ betragen.

Es hat sich als hilfreich erwiesen, die biplanare Osteotomie vor dem Sägen zunächst mit dem Elektrokauter auf der Tibiakortikalis zu markieren. Der Sägevorgang muss unter kontinuierlicher Spülung der oszillierenden Säge erfolgen.

Beim horizontalen Sägeschnitt wird lateral eine Knochenbrücke von 5-10 mm belassen, d.h. die Gegenkortikalis wird nicht durchtrennt.

Bei der ventralen ansteigenden Osteotomie wird jedoch eine komplette Osteotomie einschließlich einer Durchtrennung der Gegenkortikalis durchgeführt. Anschließend wird die Osteotomie durch vorsichtiges Einbringen mehrerer Flachmeißel in den Osteotomiespalt langsam

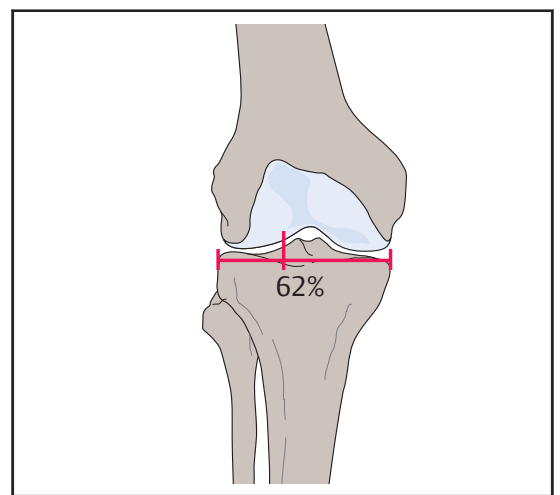

Abb.9 Die mechanische Tragachse schneidet nach Korrektur die Tibiaplateaulinie im lateralen Kompartiment im 62\%-Punkt (aus: Lobenhoffer P, Agneskirchner JD, Galla M. Kniegelenknahe Osteotomien mit Plattenfixateuren - Indikation, Planung und Operationstechnik. Stuttgart: Thieme; 2006).

geöffnet (Abb.8), alternativ kann auch ein Spreizmeißel eingesetzt werden, der langsam geöffnet wird. Die Öffnung des Osteotomiespalts wird beim Aufspreizvorgang immer wieder mit einem Messkeil oder einem Lineal überprüft. Nachdem die präoperativ geplante Öffnungshöhe erreicht ist, wird in die posteromediale Kortikalisecke der Osteotomie ein Arthrodesenspreizer eingesetzt. Das Bein wird anschließend vorsichtig in Streckung gebracht und die mechanische Beinachse wird klinisch und radiologisch kontrolliert. Hierzu kann eine Messstange verwendet werden, die unter Durchleuchtungskontrolle über dem Hüftkopfzentrum und dem Zentrum des oberen Sprunggelenks platziert wird. Die Verwendung eines Elektrokauterkabels hat sich hier als ungenau erwiesen.



Abb. 10 Einschieben des Plattenfixateurs mit den vormontierten Bohrbüchsen und Distanzstopfen in einen Subkutantunnel.

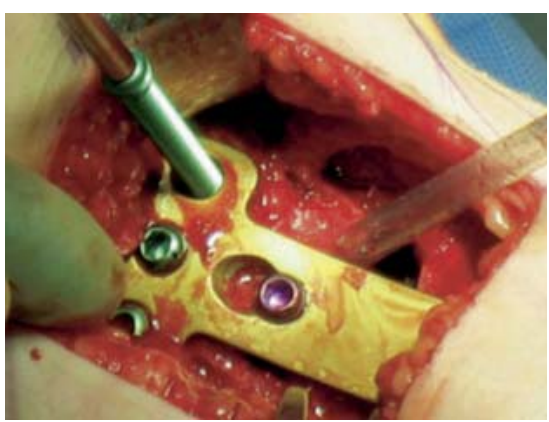

Abb. 11 Proximale Verriegelung mit selbstschneidenden Kopfverriegelungsschrauben.

Die Traglinie sollte die Tibiaplateaulinie im 62\%-Punkt im lateralen Kompartiment schneiden [4] (Abb. 9) und kann ggf. durch weiteres Öffnen oder Schließen des Arthrodesenspreizers korrigiert werden. Darüber hinaus muss die Neigung der tibialen Gelenkfläche in der Sagittalebene (engl.: „tibial slope“) beachtet werden.

Diese sollte sich bei der Korrektur einer reinen Varusdeformität nicht verändern!

Ist das gewünschte Korrekturergebnis erreicht, wird der Plattenfixateur mit den vormontierten Distanzstopfen und proximalen Bohrhülsen in einen präparierten Subkutantunnel eingeschoben (Abb. 10). Der lange Plattenschenkel wird auf dem Tibiaschaft ausgerichtet, sodass er weder ventral noch dorsal die Kortikalis überragt und der nicht durchbohrte Mittelteil des Fixateurs genau über dem Osteotomiespalt zu liegen kommt. Zunächst werden die selbstschneidenden Kopfverriegelungsschrauben in den 3 proximalen Plattenlöchern im T-Schenkel des Implantats eingebracht (Abb. 11). Anschließend wird das 1. Plattenloch distal der Osteotomie mit einer temporären Zugschraube besetzt. Hierdurch wird der Plattenfixateur an das distale Osteotomiesegment heran- 
gezogen und die laterale Kortikalisbrücke gelangt unter Kompression (Abb. 12). Beim Eindrehen dieser Schraube muss darauf geachtet werden, dass sich die Höhe des geöffneten Osteotomiespalts nicht verändert. Von distal nach proximal werden nun nacheinander die übrigen Plattenlöcher über eine kleine Stichinzision mit monokortikalen selbstschneidenden/selbstbohrenden Kopfverriegelungsschrauben besetzt (Abb. 13). Die vormontierten Distanzstopfen werden erst unmittelbar vor dem Bohren aus dem jeweiligen Loch entfernt. Im letzten Schritt wird die Zugschraube im 1. Plattenloch distal der Osteotomie durch eine bikortikale Verriegelungsschraube ersetzt. Der Arthrodesenspreizer kann nun entfernt werden.

Bei intakter lateraler Knochenbrücke und gutem Knochenkontakt im anterioren aufsteigenden Osteotomieschenkel kann regelhaft auf eine Spongiosaplastik und auf das Einbringen von Knochensubstituten verzichtet werden. Bei osteoporotischen Knochenverhältnissen oder problematischer Knochenheilung (Nikotinabusus) empfiehlt sich das Auffüllen des Osteotomiespalts mit autologer Spongiosa aus dem vorderen Beckenkamm.

Die Implantation von kortikospongiösen Spänen ist angesichts der hohen mechanischen Festigkeit des Implantats nicht erforderlich. Über den Osteotomiespalt kann ein Kollagenvlies gelegt werden. Eine Überlaufdränage wird eingelegt und nach proximal ausgeleitet, eine Saugdränage ist nicht notwendig. Nach abschließender radiologischer Dokumentation in 2 Ebenen (Abb. 14) wird die Operation durch schichtweisen Wundverschluss beendet.

\section{Nachbehandlung}

Der Verbandswechsel erfolgt am 1. postoperativen Tag. Da sich nach der Operation oftmals ein ausgeprägtes Lymphödem am Unterschenkel bildet, müssen die Weichteilverhältnisse täglich sorgfältig beurteilt werden. Manuelle Lymphdränage sollte im Wechsel mit Kryotherapie ab dem 1. postoperativen Tag täglich durchgeführt werden. Sehr bewährt hat sich der Einsatz einer automatischen intermittierenden Venenkompressionspumpe.

Die Mobilisation beginnt ab dem 1. Tag an Unterarmgehstützen unter Teilbelastung mit 15 kg Körpergewicht.
Nach Wundheilung und Wiederherstellung der muskulären Koordination kann die Belastung schrittweise schmerzadaptiert gesteigert werden, sodass in den meisten Fällen nach 4 Wochen voll belastet werden kann.

Das Bewegungsausmaß des Kniegelenks muss nicht limitiert werden, sodass eine Orthesenversorgung in der Regel nicht notwendig ist.

Bis zum vollständigen Erreichen der Vollbelastung sollte die medikamentöse Thromboembolieprophylaxe mit niedermolekularem Heparin unter regelmäßiger Kontrolle der Thrombozytenzahl durchgeführt werden. Die krankengymnastische Nachbehandlung umfasst sowohl aktive als auch passive Bewegungsübungen. Eine Elektrotherapie zur Muskelstimulation (EMS-Gerät) kann eingesetzt werden. Die Hautfäden werden am 10.-12. postoperativen Tag entfernt. Die Röntgenkontrollen des Kniegelenks mit proximalem Unterschenkel in 2 Ebenen erfolgen in der 1. postoperativen Woche und in der 4. postoperativen Woche vor dem Übergang zur Vollbelastung. Zur postoperativen Beurteilung der mechanischen Tragachse kann zusätzlich eine Ganzbeinaufnahme im Stehen angefertigt werden.

Die Implantatentfernung sollte frühestens nach 1 Jahr vorgenommen werden.

\section{Fehlermöglichkeiten und Komplikationen}

Fehler können durch genaue Kenntnis des Implantats und eine sorgfältige präoperative Planung vermieden werden.

Die winkelstabile Verriegelung muss ohne Verkanten des Schraubenkopfs im Gewinde erfolgen, die durch die Plattenlöcher vorgegebene Verlaufsrichtung der Kopfverriegelungsschrauben muss genau beachtet werden.

Darüber hinaus muss auf die korrekte Positionierung des Plattenfixateurs geachtet werden. Der lange Plattenschenkel muss genau am Tibiaschaft ausgerichtet werden und der proximale Anteil muss so platziert werden, dass ausreichend Platz für alle proximalen Verriegelungsschrauben des T-Schenkels gegeben ist. Bei sorgfältiger präoperativer Planung und intraoperativer Überprüfung der Beinachse kann eine Über- oder Unterkorrektur ausgeschlossen werden. Das Risiko einer Verletzung der großen Gefäßnervenbahnen beim Sägen der poste-



Abb. 12 Besetzen des 1. Plattenlochs distal der Osteotomie mit einer temporären Zugschraube (Schraubendreher), um Kompression zu erzeugen (aus: Lobenhoffer P, Agneskirchner JD, Galla M. Kniegelenknahe Osteotomien mit Plattenfixateuren - Indikation, Planung und Operationstechnik. Stuttgart: Thieme; 2006).



Abb. 13 Distale Verriegelung mit monokortikalen selbstschneidenden/selbstbohrenden Kopfverriegelungsschrauben über kleine Stichinzision.

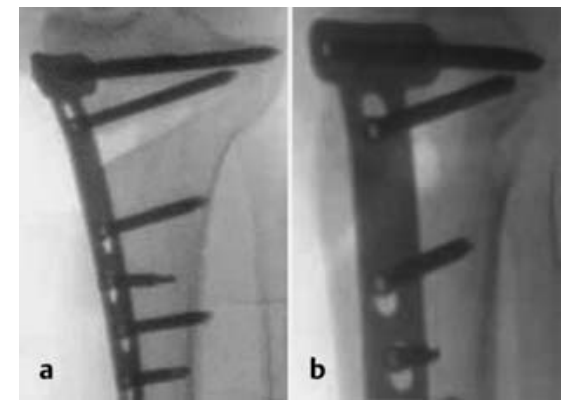

Abb. 14a und b Abschließende radiologische Dokumentation des Operationsergebnisses unter Bildwandlerkontrolle in beiden Ebenen.

rioren Tibiakortikalis kann durch den Einsatz schützender Hohmann-Haken vermieden werden.

Die häufig auftretende postoperative Weichteilschwellung und das Lymphödem können medikamentös durch orale antiphlogistische Therapie und durch physikalische Behandlungen mit manu- 
eller Lymphdränage behandelt werden (s.o.).

Eine tiefe Beinvenenthrombose und/ oder eine Lungenembolie muss mit einer entsprechenden Heparintherapie und überlappender Einleitung einer Marcumartherapie behandelt werden.

Die klinischen Symptome eines drohenden Kompartmentsyndroms (prallelastische Schwellung der Extremität und Sensibilitätsstörungen am Unterschenkel) müssen rechtzeitig erkannt werden. Der tolerable Kompartmentdruck richtet sich v.a. nach der klinischen Symptomatik. Der Kompartmentdruck muss ggf. mit einem Kompartmentmessgerät kontrolliert werden. Beträgt die Differenz zwischen dem gemessenen Wert und dem diastolischen Blutdruck weniger als $30 \mathrm{mmHg}$, ist eine umgehende Faszienspaltung erforderlich.

Bei einer ausgedehnten Hämatombildung ist eine Ausräumung und Dränage erforderlich. Ein postoperativer Infekt muss mit einem Débridement revidiert werden. Die systemische Antibiose ist obligat, ggf. können auch Antibiotikaträgersubstanzen in situ eingebracht werden. Ist die Osteosynthese stabil und der Weichteilmantel erhalten, kann das Implantat belassen werden. Andernfalls ist ein Verfahrenswechsel auf einen externen Fixateur zu empfehlen.

Eine verzögerte knöcherne Heilung äußert sich oftmals durch anhaltende Belastungsschmerzen nach der 6 . bis 8 . postoperativen Woche. In der a.-p. Röntgenaufnahme ist bei diesen Patienten meist ein kleiner Kallus an der lateralen Kortikalis der Osteotomie erkennbar. In diesen Fällen sollte eine sekundäre Spongiosaplastik durchgeführt werden.

\section{Ergebnisse}

Im Rahmen einer Multicenterstudie wurden 538 konsekutive Patienten aus 3 Zentren dokumentiert, welche in den Jahren 2004 bis 2006 operiert wurden. 391 Patienten konnten 3-5 Jahre postoperativ mittels standardisiertem Telefoninterview nachuntersucht werden.
Das mittlere Alter betrug 49 Jahre, 69\% waren Männer. 85\% der Patienten hatten Knorpelschäden im medialen Kompartiment Grad III oder IV nach Outerbridge (freiliegende Subchondralschicht). Die mittlere Osteotomieöffnung betrug $10 \mathrm{~mm}$. In 8 Fällen war bei verzögerter Heilung sekundär eine Spongiosaplastik nötig, 1-mal kam es zu einem Plattenbruch. 33 Patienten entwickelten Hämatome oder Wundheilungsstörungen, die revidiert wurden. In 9 Fällen wurde im Nachuntersuchungszeitraum eine Knieprothese implantiert.

Als Parameter für das funktionelle Resultat wurde der Oxford-Kniescore verwendet. Dieser Score ist in der Endoprothetik sehr verbreitet und wurde ins Deutsche übersetzt und validiert. Es handelt sich um einen subjektiven Outcome-Score, der in 12 Fragen die Belastungsfähigkeit des Kniegelenks unter Alltagsbedingungen abfragt. Im besten Fall kann der Patient 48 Punkte erreichen, im schlechtesten Fall 0 Punkte. Der mittlere Wert für den Oxford-Score betrug nach 3-5 Jahren 40 Punkte (SD 8,7). Weder in den höheren Altersgruppen noch in den Gruppen mit fortgeschrittener medialer Arthrose unterschied sich der Score signifikant vom Gesamtkollektiv. Das funktionelle Outcome nach HTO entsprach somit jenem, welcher nach unikondylärer Knieprothese erwartet wird und war besser als jenes, das nach Totalprothese des Kniegelenks verzeichnet wird.

\section{Schlussfolgerung}

Die beschriebene OP-Technik der medial öffnenden Osteotomie der proximalen Tibia ist ein geeignetes Verfahren zur Korrektur einer Varusdeformität der unteren Extremität. Die Vorteile dieser Vorgehensweise im Vergleich zur lateral schließenden Technik sind die Vermeidung einer Beinverkürzung und einer Schädigung des N. peronaeus superficialis. Die Extensorenmuskulatur und das proximale Tibiofibulargelenk müssen nicht gelöst werden. Eine Nachkorrektur der Beinachse (sog. „fine-tuning“) ist intraoperativ problemlos durch weiteres Öffnen oder Schließen des Osteotomiespalts möglich. Ligamentäre Instabilitä- ten des Kniegelenks können zeitgleich durch Veränderung der Neigung des Tibiaplateaus in der Sagittalebene („tibial slope") behandelt werden. Die laterale Knochenbrücke und das winkelstabile Implantat gewährleisten eine hohe biomechanische Primärstabilität, die eine rasche Mobilisation des Patienten erlaubt.

\section{Literatur}

${ }^{1}$ Aydogdu S, Cullu E, Arac $N$ et al. Prolonged peroneal nerve dysfunction after high tibial osteotomy: pre- and postoperative electrophysiological study. Knee Surg Sports Traumatol Arthros 2000; 8: 305-308

2 Coventry $M B$. Upper tibial Ostoetomy. Clin Orthop Relat Res 1984; 182: 46-52

3 Fujisawa Y, Masuhara K, Shiomi S. The effect of high tibial osteotomy on osteoarthritis of the knee. An arthroscopic study of 54 knee joints. Orthop Clin North Am 1979; 10: 585608

${ }^{4}$ Lobenhoffer P, De Simoni C, Staubli AE. Opening wedge high-tibial osteotomy with rigid plate fixation. Tech Knee Surg 2002; 1: 93105

5 Outerbridge RE. The Etiology of chondromalcia Patellae. J Bone Joint Surg [Br] 1961; 43 : 752-757

\section{Weiterführende Literatur}

Lobenhoffer $P$, Agneskirchner JD, Galla $M$. Kniegelenknahe Osteotomien mit Plattenfixateuren - Indikation, Planung und Operationstechnik. Stuttgart: Thieme; 2006 Lobenhoffer $P$, von Heerwaerden R, Staubli A, Jakob $R$. Osteotomies around the knee. AO Publishing. Stuttgart: Thieme; 2008

\section{Dr. Mellany Galla}

Ärztin für Orthopädie und Unfallchirurgie

Chirurgie im Medicinum

Goslarsche Landstraße 19

31135 Hildesheim

melgalla@web.de

Prof. Dr. Philipp Lobenhoffer

Arzt für Orthopädie und Unfallchirurgie

Sportsclinic Germany

Uhlemeyerstraße 16

30175 Hannover

philipp.lobenhoffer@

sportsclinicgermany.com 